\title{
Vigilancia de la publicidad de productos químicos para piscinas comercializados por internet
}

\section{Advertisement surveillance for swimming pool chemicals commercialized over the Internet}

\author{
Joaquín Gámez de la Hoza, Ana Padilla Fortes ${ }^{b}$, Marta Padilla-Ruizc \\ a Distrito sanitario costa del sol, Servicio de Salud Pública, Servicio Andaluz de Salud, España \\ b Distrito sanitario Málaga, Unidad de Prevención de Riesgos Laborales, Servicio Andaluz de Salud, España \\ c Department of Geomatics Engineering, University of Calgary, Canada
}

\section{Resumen}

Introducción: La comunicación de los peligros asociados a los productos químicos es un aspecto fundamental para mejorar la confianza de los consumidores en el comercio electrónico. Objetivo: Examinar las indicaciones de peligro reglamentarias asociadas con productos químicos para piscinas puestos a disposición de terceros a través de su publicidad por internet. Metodología: Se seleccionaron aleatoriamente 40 productos químicos peligrosos de piscinas comercializados en tiendas online a través de internet procedentes de 8 empresas con sede social en Andalucía. Resultados: Predominaron los productos sin indicaciones de peligro visibles junto a su publicidad $(n=25)$. Las indicaciones de peligros en las etiquetas de los productos en la web resultaron ilegibles. Únicamente 3 productos mostraron los códigos y frases de peligro visibles en el sitio web. Conclusión: La protección de consumidores y usuarios de comercio electrónico en materia de seguridad química puede verse comprometida debido a la falta de información relevante sobre la peligrosidad de los productos comercializados a través de internet.

Palabras clave: Comunicación en salud; productos químicos peligrosos; etiquetado de productos; publicidad; seguridad; piscinas; comercio; salud pública.

\section{Abstract}

Introduction: Communication of hazards related to chemical products is an essential aspect for improving customer confidence in electronic commerce. Objective: To examine the regulated hazard statements for swimming pool chemicals advertised and sold to the public over the Internet. Methodology: We randomly selected 40 hazardous chemicals for swimming pools sold in online stores over the Internet. Data were captured from 8 different companies' websites with headquarters in Andalusia (Spain). Results: Chemical advertisements without visible hazard statements were predominant $(n=25)$. In all cases, hazard statements on product labels displayed on the websites were illegible. Only 3 chemical products showed codes and visible hazard statements on their website. Conclusion: The protection of e-commerce consumers and customers concerning chemical safety could be compromised due to the lack of relevant information about selling hazardous products over the Internet.

Keywords: Health communication; hazardous chemicals; product labelling; advertisement; safety; swimming pools; commerce; public health. 


\section{Introducción}

$\mathrm{E}$ xiste una creciente preocupación relacionada con los peligros asociados a los productos químicos. El sector de piscinas es uno de los principales que incluye a usuarios finales de productos químicos. Los compuestos utilizados para asegurar la calidad sanitaria del agua de baño comprenden mezclas y/o sustancias químicas con diferentes grados de peligrosidad para la salud humana, desde productos nocivos, corrosivos e irritantes hasta los tóxicos para el medio ambiente. En el ámbito del reglamento europeo 528/2012, relativo la comercialización y el uso de los biocidas (UE, 2012), se han registrado en España más de 2.000 productos químicos para el tratamiento del agua de piscinas con uso autorizado tanto para el público en general como para personal profesional. Estas cantidades integran productos químicos del tipo 2 que incluye fundamentalmente bactericidas y alguicidas. Los compuestos más habituales son el cloro en forma de hipoclorito y otros derivados (ej. cloroisocianuratos), y ácidos como el clorhídrico o el sulfúrico.

Una de las tareas más importantes en salud pública es la protección de consumidores y usuarios frente a los productos químicos peligrosos. Se han notificado diversos problemas de salud asociados con la exposición a productos químicos en piscinas (Anderson et al., 2014; Centro para el Control y la Prevención de Enfermedades, 2009; Thomas y Murray, 2008), muchos de ellos debido a una manipulación incorrecta, aunque otros como consecuencia de una comunicación inadecuada sobre los peligros asociados. A veces las sustancias y mezclas químicas utilizadas no siempre son etiquetadas correctamente (Tsai et al., 2016). Para el público, la etiqueta del envase es la información inmediata que muestra la clasificación de peligrosidad del producto y las medidas de seguridad recomendadas para su uso y manipulación en el estado en que se comercializa. Adicionalmente, la ficha de datos de seguridad (FDS) está orientada al usuario profesional como herramienta clave para poder clasificar y etiquetar correctamente aquellos productos químicos que han sido formulados a partir de otros.

Al margen del presumible uso del etiquetado y FDS como fuentes de información, se han detectado serios problemas cuando se utilizan como herramientas de comunicación del riesgo. Algunos investigadores han revelado que sólo un grupo muy reducido de usuarios atienden al contenido de las etiquetas (Ferrell et al., 1994; Kovacs et al., 1997), que además suelen tener un impacto limitado en materia de seguridad (McGrath, 2011). Incluso los profesionales pueden no entender el significado de los pictogramas de peligrosidad presentes en las etiquetas (Martí Fernández et al., 2015; Hara et al., 2007). Igualmente, la FDS contiene información técnica que puede ser incomprensible para el público y los usuarios profesionales (Seki et al., 2001; Phillips et al., 1999). Estos hallazgos ponen de manifiesto que la efectividad de las advertencias de peligros requiere que sean visibles y comprensibles por cualquier tipo de usuario.

El notable desarrollo normativo experimentado en materia de seguridad química ha estado en gran parte motivado por el impacto de los productos químicos en la salud pública. La importancia sanitaria de la regulación de medidas preventivas radica en garantizar la protección de la salud humana y del medio ambiente, así como la seguridad de los usuarios que entran en contacto con productos químicos peligrosos. En este contexto, el reglamento europeo REACH (Registration, Evaluation, Authorisation and Restricción of Chemicals) establece que deben comunicarse los peligros asociados a los productos químicos comercializados (UE, 2006), mientras que el reglamento CLP (Classification, Labelling and Packaging) pretende aplicar criterios armonizados de clasificación y etiquetado (UE, 2008).

Sin embargo, el protagonismo alcanzado por las nuevas tecnologías hace que el acceso a Internet posibilite a los consumidores adquirir productos químicos que se suministran y entregan, a menudo traspasando las fronteras entre países, sin interactuar cara a cara con un profesional del sector (UE, 2000). Entre las potenciales amenazas de la venta online cabe mencionar la presencia de productos clandestinos (sin registro oficial), formulaciones de escasa calidad (elaboraciones artesanales), deficiencias de las condiciones de almacenamiento durante el suministro, sistemas de información erróneos e incompletos y carencia de un soporte profesional efectivo. Estos inconvenientes hacen que la protección de los consumidores y el control de los sitios web que venden productos químicos, tanto a nivel nacional como internacional, sea un proceso complejo y difícil. 
En efecto, muchos de los problemas en las relaciones entre proveedor y consumidor provienen del desconocimiento de los peligros asociados a lo que se está adquiriendo o usando. En este sentido es esencial que tanto los profesionales como cualquier usuario de productos de piscinas puedan acceder a información que sea correcta y les permita dotarse de criterios apropiados para la utilización segura de los productos químicos.

Con el propósito de concienciar a los agentes de la cadena de suministro y sensibilizar a las empresas del sector y al consumidor general, nuestro objetivo fue examinar las indicaciones de peligro reglamentarias asociadas a productos químicos para piscinas puestos a disposición de terceros a través de su publicidad por internet. Un objetivo secundario ha sido identificar los pictogramas de peligro e indicaciones de advertencia presentes en el etiquetado del producto químico ofrecido en el sitio web utilizado para su venta.

\section{Metodología}

\section{Diseño del estudio}

Análisis descriptivo con diseño observacional. La unidad de análisis fue cada uno de los sitios web de comercio electrónico de productos químicos para piscinas, con emplazamiento en la comunidad autónoma de Andalucía. Como fuente de recuperación de datos se utilizó el motor de búsqueda Google. El acceso al buscador se realizó durante el mes de marzo del 2018, utilizando su herramienta "búsqueda de lugares" que ofrece una lista detallada de establecimientos agrupados sobre lugares específicos en función de las palabras y comandos empleados en el campo de búsqueda. El criterio de búsqueda de los sitios web con publicidad sobre productos químicos comercializados se basó en el uso de la cadena de búsqueda: productos químicos piscinas AND "nombre de cada provincia andaluza". A los efectos de esta investigación, se estableció que la publicidad comprende una forma especial de comercialización que permite la puesta a disposición de un producto químico a un consumidor por medio de pedidos online (comercio por internet) y realizar un contrato de compraventa a distancia.

\section{Criterios de inclusión}

El perfil preferente de los participantes fue los sitios web de proveedores de productos químicos para piscinas con tiendas online (disponibilidad de carro de compra en la propia página web). Cuando no se encontró ese perfil, se amplió la búsqueda a proveedores profesionales de grandes superficies. Como resultado de la búsqueda se seleccionaron aleatoriamente un total de 40 productos químicos peligrosos objeto de estudio, correspondientes a 5 por empresa con sede física y/o social localizada una por cada una de las 8 provincias andaluzas. De cada sitio web se priorizaron productos (mezclas químicas) etiquetados en castellano, clasificados como peligrosos y accesibles al público en general. La selección de productos fue por orden de aparición, rechazando coincidencia de productos del mismo fabricante.

Se descartó la publicidad de pequeña escala que no ofrezca opción de compra (ej. banners). Tampoco fue objeto de evaluación las normas precontractuales mínimas que rigen entre vendedor y comprador reguladas conforme a los derechos de protección de los consumidores en materia de contratos a distancia.

\section{Mediciones principales}

Al objeto de verificar si se cumple con unos requisitos básicos de comunicación del riesgo se comprobó si la publicidad del producto químico mencionaba las indicaciones de peligro (Frases $\mathrm{H}$ ) e información suplementaria (Frases EUH) previstas en el reglamento CLP (UE, 2008), ya sea en forma de texto escrito directamente en la propia página web o bien con la imagen clara de una etiqueta que sea fácilmente legible. Adicionalmente, se verificó si contenían pictogramas de peligros y palabras de advertencia que avisen al lector/ comprador de un peligro potencial.

Se efectuó un análisis cuantitativo y se confeccionaron diferentes presentaciones gráficas utilizando el software Microsoft Excel 2016 v.16 para Windows 10. Para el control de calidad de la información se realizó una revisión por pares, corrigiendo posibles errores en la introducción de datos.

\section{Resultados}

La investigación reveló que, en nuestra unidad de estudio y emplazamiento, el segmento de mercado de piscinas con tiendas online de productos químicos estuvo formado principalmente por microempresas comercializadoras de ámbito nacional. Generalmente las empresas no ostentaban la titularidad de los productos químicos, aunque tres de ellas eran fabricantes en una 
gama limitada comercializada. La tabla 1 muestra la descripción de las características socio-económicas de las empresas seleccionadas.

\section{Tipología y peligrosidad de los productos}

Observamos que mayoritariamente la presentación comercial de los productos químicos envasados era en estado líquido $(n=28)$, el resto en estado sólido $(n=12)$, y su función principal fue como agentes biocidas $(n=28)$ -es decir, bactericidas y alguicidas destinados a destruir, contrarrestar, neutralizar, impedir la acción o ejercer un control de otro tipo sobre cualquier organismo nocivo presente en el agua de baño-. En la composición de las mezclas químicas identificadas intervinieron sustancias que confieren la peligrosidad al producto comercializado, dependiendo de la materia activa y su concentración. Las sustancias activas identificadas más relevantes fueron el ácido tricloroisocianúrico y sales de amonio cuaternario.

Atendiendo a las propiedades fisicoquímicas del producto final, la categoría de peligro dominante fue de tipo comburente $(n=9)$, mientras que por sus efectos en la salud humana fue corrosivo $(n=25)$. Con respecto a los daños al medio ambiente, más de la mitad de los productos se clasificaron como tóxicos para el medio ambiente acuático $(n=22)$. El resumen de los productos químicos reconocidos conforme a su composición, finalidad y categoría de peligrosidad se muestra en la tabla 2. No se encontró información sobre restricciones de uso de los productos comercializados para el público general.

Tabla 1. Características socio-económicas de las empresas participantes $(n=8)$

\begin{tabular}{|l|c|}
\hline & $\mathbf{n}$ \\
\hline Sede social & 8 \\
Identificación del titular & \\
\hline Tipo de empresa & 7 \\
Proveedor profesional & 1 \\
Gran superficie & \\
\hline Tamaño de la empresa & 6 \\
$<10$ empleados & 1 \\
Entre 10-50 empleados & 1 \\
$>$ 250 empleados & \\
\hline Clase de actividad & 8 \\
Comercialización & 3 \\
Fabricación & 1 \\
Almacenamiento & \\
\hline Nivel de mercado & 7 \\
Nacional & 1 \\
Internacional & \\
\hline
\end{tabular}

Fuente: elaboración propia 
Tabla 2. Clases de peligro y nivel de peligrosidad asociados con los compuestos químicos para piscinas investigados, agrupados según su finalidad $(n=40)$.

\begin{tabular}{|c|c|c|c|c|c|}
\hline & \multirow{2}{*}{\begin{tabular}{|c|} 
Físico \\
Comburente
\end{tabular}} & \multicolumn{3}{|c|}{ Salud humana } & \multirow{2}{*}{$\begin{array}{c}\text { Ambiental } \\
\text { Peligroso para el } \\
\text { medioambiente }\end{array}$} \\
\hline & & Nocivo & Corrosivo & Irritante & \\
\hline \multicolumn{6}{|l|}{ Bactericidas } \\
\hline Hipoclorito de sodio & & & 3 & & 2 \\
\hline Ácido tricloroisocianúrico & 5 & 4 & & 5 & 5 \\
\hline $\begin{array}{l}\text { Ácido tricloroisocianúrico +sulfato de } \\
\text { cobre }\end{array}$ & 2 & 1 & 1 & 2 & 2 \\
\hline Bromocloro-dimetilhidantoina & 2 & 1 & 2 & 1 & 2 \\
\hline Peroximonosultafo de pentapotasio & 1 & 1 & 1 & 1 & 1 \\
\hline Dicloroisocianurato de sodio & & & & 1 & 1 \\
\hline Peróxido de hidrógeno & & 1 & 1 & & \\
\hline \multicolumn{6}{|l|}{ Alguicidas } \\
\hline Amonio cuaternario, cloruros & & & 4 & 3 & 8 \\
\hline Amonio cuaternario +sultafo de cobre & & 1 & 1 & & 2 \\
\hline $\begin{array}{l}\text { Ácido tricloroisocianúrico + sulfato de } \\
\text { cobre }\end{array}$ & 2 & 1 & 1 & 1 & 2 \\
\hline Sulfato de cobre & & 1 & & & 1 \\
\hline Ácido tricloroisocianurico +ácido bórico & 1 & 1 & & 1 & 1 \\
\hline Peroximonosultafo de pentapotasio & 1 & 1 & 1 & 1 & 1 \\
\hline Bromocloro-dimetilhidantoina & 1 & & 1 & & 1 \\
\hline Dicloroisocianurato de sodio & & & & 1 & 1 \\
\hline \multicolumn{6}{|l|}{ Modificadores del pH } \\
\hline Ácido Clorhídrico & & & 4 & 1 & \\
\hline Hidróxido de sodio & & & 2 & & \\
\hline \multicolumn{6}{|l|}{ Floculantes } \\
\hline $\begin{array}{l}\text { Ácido tricloroisocianúrico +sulfato de } \\
\text { cobre }\end{array}$ & 2 & 1 & 1 & 1 & 2 \\
\hline Polihidroxicloruro de aluminio & & & 1 & 1 & \\
\hline \multicolumn{6}{|l|}{ Limpiadores de superficies } \\
\hline Ácido Clorhídrico & & & 2 & & \\
\hline Amonio cuaternario, cloruros & & & 1 & & \\
\hline Sin especificar & & & 2 & & 1 \\
\hline \multicolumn{6}{|l|}{ Desinfectante de superficies } \\
\hline Sulfato de cobre & & 1 & & & 1 \\
\hline
\end{tabular}




\section{Comunicación de peligros en la web}

Mayoritariamente los productos químicos puestos a la venta a través de internet no llevaban asociadas indicaciones de peligro directamente visibles junto a su publicidad $(n=25)$. De las 8 empresas participantes, sólo una mostró las preceptivas indicaciones de peligro (frases $\mathrm{H}$ ) en 3 de los productos publicitados para su compraventa online. Un sitio web aportó la descripción y códigos de peligro (frases $\mathrm{H} / \mathrm{EUH}$ ) en 5 productos a través de un enlace a una ficha técnica comercial. Asimismo, se identificaron 7 productos con enlaces a FDS en ficheros descargables que contenían las frases de peligro tanto de la mezcla final como de los ingredientes clasificados como peligrosos. Seis de las FDS tenían un desfase de 5 años desde su última revisión. En la figura 1 se presenta la localización de las indicaciones de peligro asociadas a la publicidad de los productos químicos comercializados a través de las tiendas online de los proveedores investigados.

\section{Etiquetado}

Las etiquetas de los productos químicos se exhibieron en todas las tiendas online visitadas, aunque su contenido fue incompleto (Figura 2). En 19 productos, el contenido de las etiquetas visualizadas se limitaba a mostrar la denominación comercial acompañada generalmente de la forma física de la mezcla química (líquido, granulado, en polvo y tabletas) y de la cantidad nominal (masa o volumen). Mientras que, en los 21 restantes, se presentó una imagen del etiquetado completo, pero sin que las frases de riesgo e indicaciones de peligro fuesen visibles y de fácil lectura, ni tan siquiera con la opción de zoom de la imagen.

La única señal visible de advertencia para el consumidor o lector del sitio web fue la presencia del símbolo normalizado de peligro en 21 de las etiquetas de los envases de productos químicos, aunque en 4 casos carecían de la palabra que describe el tipo de peligro asociado al símbolo y que acompañan al pictograma. Asimismo, se detectó que únicamente 9 pictogramas estaban actualizados al nuevo sistema globalmente armonizado (GHS, Global Harmonized System) de clasificación y etiquetado de productos químicos adoptado por el reglamento europeo CLP (Tabla 3).

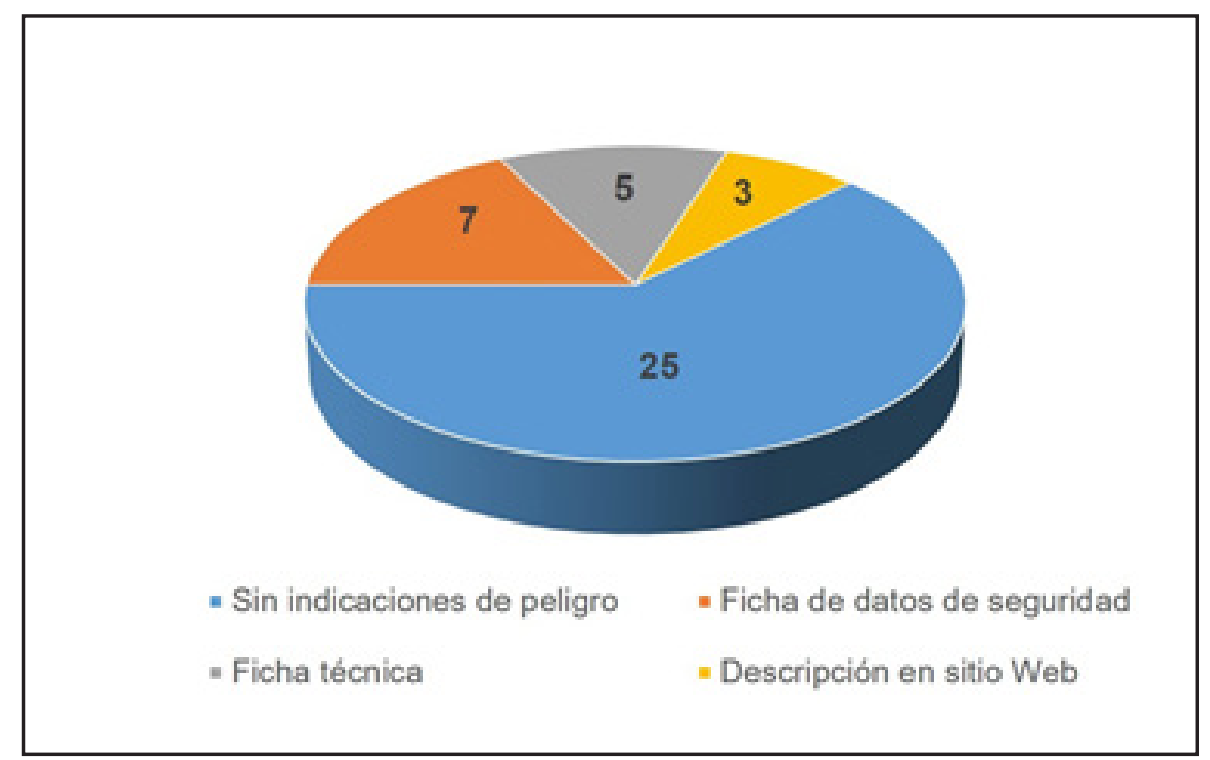

Figura 1. Localización de frases de peligro en los productos químicos de piscinas investigados (n=40). 


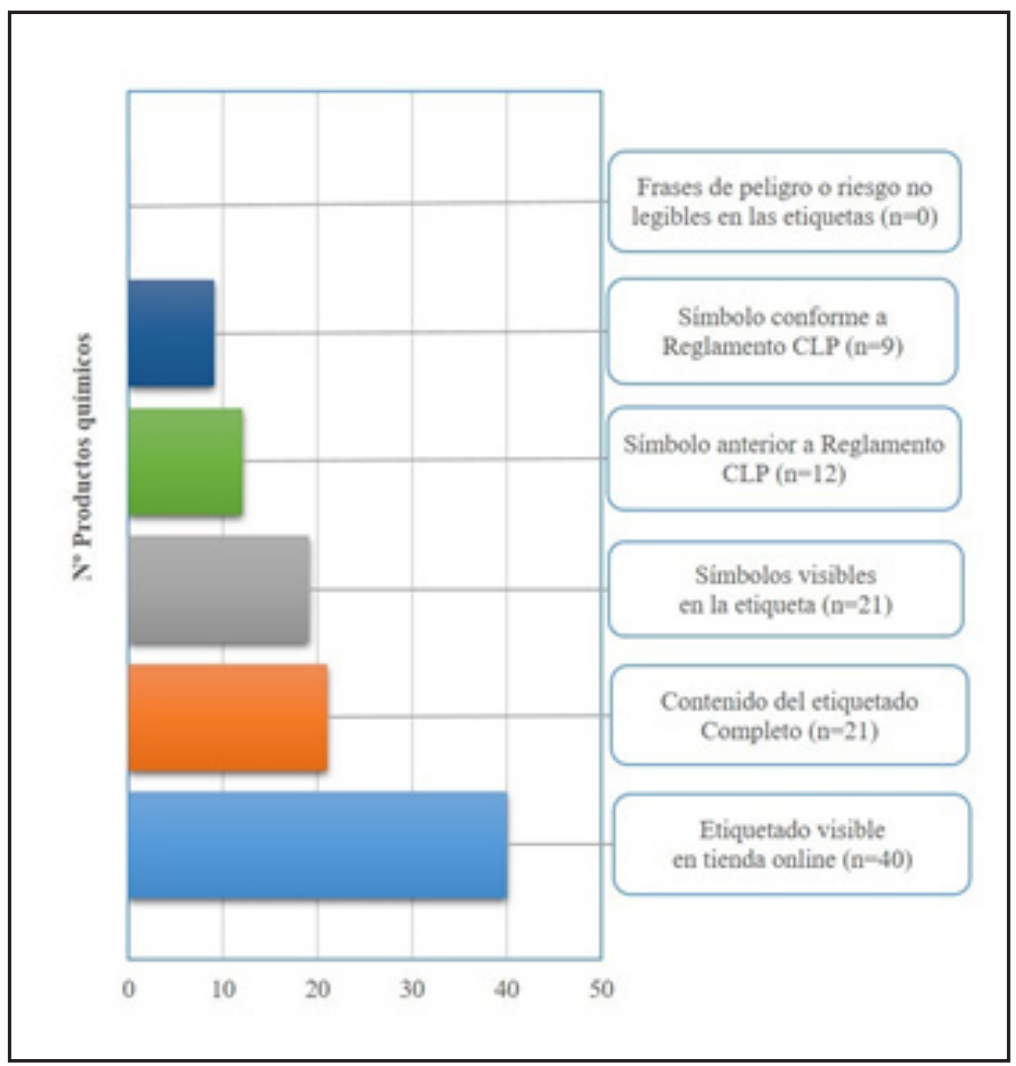

Figura 2. Indicaciones de peligro en el etiquetado de los productos químicos para piscinas investigados en páginas Web $(n=40)$.

Tabla 3. Muestra de pictogramas de peligrosidad identificados en el etiquetado de los productos químicos de piscinas investigados en páginas Web $(n=40)$.

\begin{tabular}{|l|l|l|l|l|l|}
\hline Clase de peligro & Comburente & Corrosivo & Nocivo & Irritante & $\begin{array}{c}\text { Peligroso } \\
\text { para el medio } \\
\text { ambiente }\end{array}$ \\
\hline $\begin{array}{l}\text { Conforme al } \\
\text { Reglamento CLP }\end{array}$ & & & &
\end{tabular}

* Equivalencias según Anexo VII del Reglamento CLP 


\section{Discusión}

Los resultados de esta investigación ponen de manifiesto que la comunicación de los peligros asociados con los productos químicos para piscinas puestos a la venta a través de internetfue inadecuada. Nos encontramos con un comercio electrónico donde casi la mitad de los productos investigados carecian de indicaciones de peligro junto a su publicidad en la web. Las advertencias explícitas aumentan la percepción del riesgo relacionada con los productos peligrosos (Heaps y Henley, 1999), facilitando que consumidores y usuarios puedan seleccionarlos conociendo al instante el nivel de peligrosidad de los productos cuando acceden a una tienda online. Por el contrario, la ausencia de indicaciones de los peligros del producto puede propiciar una falsa sensación de seguridad por ignorarse los riesgos a los que podría estar expuesto tras la compra del producto (Oldershaw y Fairhurst, 2001). En cierta medida el proveedor profesional traslada al comprador la responsabilidad de recabar información técnica, dando pocas facilidades informativas previamente a la adquisición del producto.

Es preciso que el público pueda acceder a información de seguridad completa, correcta y comprensible. Aun así, conviene tener presente que esta correcta comprensión no garantiza que los usuarios actúen de forma segura. La información genérica, como por ejemplo el modo de empleo y la finalidad del producto, puede resultar insuficiente para su aplicación segura en las piscinas. Hay que resaltar que las indicaciones de peligro están referidas al estado en que se comercializa el producto, pero no necesariamente en la forma en que se aplican (ej. diluido o depositado en skimmers perimetrales al vaso de la piscina). Por ello, es muy importante que el consumidor disponga inmediatamente de las advertencias adecuadas para saber cómo evitar los riesgos y las consecuencias de no hacerlo.

Con este trabajo destacamos que la información sobre seguridad de compuestos químicos para piscinas ofrecida en tiendas de comercio online también fue insuficiente. Aproximadamente en uno de cada tres productos comercializados las indicaciones de peligro se proporcionaron a través de enlaces a FDS y fichas técnicas en la web del proveedor. Debido a la naturaleza técnica y copiosa de estos documentos, son los profesionales quienes conocen su jerga y poseen los recursos básicos para interpretarlos acertadamente (Bernstein, 2002). En cambio, es previsible que el público carezca de las capacidades y competencias para comprender la información técnica que proporcionan. Diferentes investigaciones evidencian que tanto su complejidad como el lenguaje técnico empleado, influyen en la correcta interpretación del significado de las advertencias de peligro de las FDS y etiquetas, y esto se agrava con el nivel educativo de los usuarios de los productos químicos (Beaufils et al., 2014; Hinks et al., 2009).

Nuestro estudio reveló una pobre calidad informativa mostrada en las etiquetas de los productos químicos comercializados en la web. Hasta las etiquetas con el contenido completo, no tenían un tamaño y resolución suficiente para ser leídas sin problemas. Aun cuando la etiqueta del producto como la FDS pueda estar disponible en la entrega de la mercancía, presumiblemente los manipuladores de productos químicos de piscinas podrían exponerse a sustancias peligrosas como resultado de una inadecuada información ofrecida en origen al consumidor (Suleiman y Svendsen, 2014). En este sentido, Lesch (2008) apunta a que las etiquetas actúan como recordatorio de la información de seguridad que el usuario ha recibido anteriormente de su proveedor.

El hallazgo más destacado sobre advertencias de peligros se evidenció por medio de la visualización de símbolos de peligrosidad en las etiquetas del producto. Los símbolos son un soporte básico de transmisión visual que constituyen uno de los elementos principales del sistema de comunicación de riesgos en seguridad química. Diferentes estudios han remarcado los beneficios de incorporar pictogramas para aumentar la efectividad de la comunicación sobre seguridad (Braun y Silver, 1995; Boelhouwer, Davis, Franco-Watkins, Dorris, y Lungu, 2013) y mejorar la comprensión de las frases de peligro que acompañan al producto (Sojourner y Wogalter, 1997).

Obviamente la interpretación del significado de los símbolos requiere un cierto grado de familiaridad y puede variar en función de la clase de símbolo, según se trate de alerta, prohibición o información general (Hancock et al., 2004). Al respecto, observamos que una considerable muestra de productos presentaban símbolos no actualizados conforme al vigente sistema internacional GHS sobre información de peligros. En cualquier caso, los símbolos por sí mismos tienen un nivel de comprensión limitado, de manera que la incorporación de frases de peligro se ha revelado como una importante ayuda complementaria para identificar símbolos escasamente comprendidos, pese a que la frase no siempre es representativa del concepto de seguridad que encarna cada símbolo (Wolff y Wogalter, 1998; Radnoff, 2013).

En conclusión, la compraventa online de productos químicos peligrosos para piscinas en Andalucía tiene un nivel de fiabilidad y protección al consumidor controvertido, debido principalmente a que las indicaciones preceptivas de peligro son omitidas en los sitios web y son ilegibles en el etiquetado de los productos mostrado al público. 
Las limitaciones de este estudio tienen que ver con el escaso número de proveedores de piscinas con sede en Andalucía que gestionan tiendas online propias en internet. No se ha entrado a valorar si el contenido de la FDS y clasificación de peligrosidad de los productos químicos eran correctas o presentaban errores, lo que sin duda hubiera aportado datos cualitativos de interés.

Como recomendación general sugerimos a los consumidores y usuarios que presten especial atención a las indicaciones de peligro de los productos químicos ofertados en la web, y a tenor de las carencias detectadas sobre comunicación de riesgos, exigir al proveedor la información preceptiva de seguridad. Corresponde a los agentes de la cadena de suministro facilitar información completa y correcta de sus productos y servicios. Por su parte, los poderes públicos tienen la obligación de promover y hacer valer el derecho a la información de los consumidores y usuarios en el comercio electrónico, atendiendo a las características específicas del sector económico y adecuando las regulaciones sobre seguridad química a la sociedad digital de la información.

\section{Referencias bibliográficas}

Anderson, A., Welles, W., Drew, J., \& Orr, M. (2014). The distribution and public health consequences of releases of chemicals intended for pool use in 17 states, 2001-2009. Journal of Environmental Health, 76(9), 10-15.

Beaufils, E., Hommet, C., Brault, F., Marqué, A., Eudo, C., Vierron, E., ... Mondon, K. (2013). The effect of age and educational level on the cognitive processes used to comprehend the meaning of pictograms. Aging Clinical And Experimental Research, 26(1), 61-65.

Bernstein, J. (2002). Material safety data sheets: Are they reliable in identifying human hazards? Journal of Allergy And Clinical Immunology, 110(1), 35-38. http://dx.doi.org/10.1067/mai.2002.124891

Boelhouwer, E., Davis, J., Franco-Watkins, A., Dorris, N., \& Lungu, C. (2013). Comprehension of hazard communication: Effects of pictograms on safety data sheets and labels. Journal of Safety Research, 46, 145-155.

Braun, C., \& Silver, N. (1995). Interaction of signal word and colour on warning labels: differences in perceived hazard and behavioural compliance. Ergonomics, 38(11), 2207-2220. http://dx.doi. org/10.1080/00140139508925263
Centro para el control y la prevención de enfermedades. (2009). Pool chemical-associated health events in public and residential settings - USA, 1983-2007. Morbidity Mortal Weekly Report, 58(18), 489-493.

Directiva relativa a determinados aspectos jurídicos de los servicios de la sociedad de la información, en particular el comercio electrónico en el mercado interior 2000 (UE).

Ferrell, M., Hollon, J., Lockwood, J., \& Wangberg, J. (1994). Pesticide labels: proven protection or superficial safety? Journal Of The American Optometric Association, 65(1), 18-26.

Hancock, H., Rogers, W., Schroeder, D., \& Fisk, A. (2004). Safety symbol comprehension: Effects of symbol type, familiarity, and age. Human Factors: The Journal Of The Human Factors And Ergonomics Society, 46(2), 183-195. http://dx.doi.org/10.1518/ hfes.46.2.183.37344

Hara, K., Mori, M., Ishitake, T., Kitajima, H., Sakai, K., Nakaaki, K., \& Jonai, H. (2007). Results of recognition tests on Japanese subjects of the labels presently used in Japan and the UN-GHS labels. Journal of Occupational Health, 49(4), 260-267. http://dx.doi. org/10.1539/joh.49.260

Heaps, C., \& Henley, T. (1999). Language matters: warning considerations in hazard perception and warning comprehension. The Journal of Psychology, 133(3), 341-351. http://dx.doi. org/10.1080/00223989909599747

Hinks, J., Bush, J., Andras, J., Garratt, J., Pigott, G., Kennedy, A., \& Pless-Mulloli, T. (2009). Views on chemical safety information and influences on chemical disposal behaviour in the UK. Science of the Total Environment, 407(4), 1299-1306. http:// dx.doi.org/10.1016/j.scitotenv.2008.10.056

Kovacs, D., Small, M., Davidson, C., \& Fischhoff, B. (1997). Behavioural factors affecting exposure potential for household cleaning products. Journal of Exposure Analysis And Environmental Epidemiology, 7(4), 505-520.

Lesch, M. (2008). Warning symbols as reminders of hazards: Impact of training. Accident Analysis \& Prevention, 40(3), 1005-1012. http://dx.doi. org/10.1016/j.aap.2007.11.009

Martí Fernández, F., Van der Haar, R., López López, J., 
Portell, M., y Torner Solé, A. (2015). La comprensión de los pictogramas de peligro de productos químicos entre trabajadores del sector de limpieza. Archivos de Prevención de Riesgos Laborales, 18(2), 6671. http://dx.doi.org/10.12961/aprl.2015.18.2.03

McGrath, J. (2011). The role of equipment warning labels in the industrial workplace. International Journal of Occupational Safety And Ergonomics, 17(1), 4960. http://dx.doi.org/10.1080/10803548.2011.11076871

Oldershaw, P., \& Fairhurst, S. (2001). Sharing toxicological information on industrial chemicals. The Annals of Occupational Hygiene, 45(4), 291-294. http:// dx.doi.org/10.1016/s0003-4878(00)00090-9

Phillips, C., Wallace, B., Hamilton, C., Pursley, T., Petty, G., \& Bayne, C. (1999). The efficacy of material safety data sheets and worker acceptability. Journal of Safety Research, 30(2), 113-122.

Reglamento relativo a la comercialización y el uso de los biocidas 2012 (UE).

Reglamento sobre clasificación, etiquetado y envasado de sustancias y mezclas (CLP) 2008 (UE).

Reglamento relativo al registro, la evaluación, la autorización y la restricción de las sustancias y preparados químicos (REACH) 2006 (UE).

Seki, A., Takehara, H., Takigawa, T., Hidehira, T., Nakayama, S., \& Usami, M. (2001). Use of material safety data sheets at workplaces handling harmful substances in Okayama, Japan. Journal of Occupational Health, 43(2), 95-100. http://dx.doi. org/10.1539/joh.43.95

Sojourner, R., \& Wogalter, M. (1997). The influence of pictorials on evaluations of prescription medication instructions. Therapeutic Innovation \& Regulatory Science, 31(3), 963-972. http://dx.doi. org/10.1177/0092861159703100340

Suleiman, A., \& Svendsen, K. (2014). Are safety data sheets for cleaning products used in Norway a factor contributing to the risk of workers exposure to chemicals? International of Journal Occupational Medicine and Environmental Health, 27(5), 840853.http://dx.doi.org/10.2478/s13382-014-0302-8

Radnoff, D. (2013). Accuracy of reported flash point values on material safety data sheets and the impact on product classification. Journal of Occupational and Environmental Hygiene, 10(10), 540-546.
Thomas, H., \& Murray, V. (2008). Review of acute chemical incidents involving exposure to chlorine associated with swimming pools in England and Wales, JuneOctober 2007. Journal of Public Health, 30(4), 391-397. http://dx.doi.org/10.1093/pubmed/fdn073

Tsai, C. J., Mao, I. F., Ting, J.Y., Young. C. H., Lin, J. S., \& Li, W. L. (2016). Quality of Chemical Safety Information in Printing Industry. Annals of Occupational Hygiene, 60(3), 361-370.

Wolff, J., \& Wogalter, M. (1998). Comprehension of pictorial symbols: Effects of context and test method. Human Factors: The Journal of The Human Factors And Ergonomics Society, 40(2), 173186. http://dx.doi.org/10.1518/001872098779480433 